\title{
Study of viral nuclear transport: system based on the "Fluorescence Resonance Energy Transfer" (FRET)
} Aurélie Deroubaix*, Michael Kann and Sébastien Lainé

\author{
Address: Microbiologie cellulaire et moléculaire et pathogénicité, UMR 5234, Université Bordeaux 2, France
}

* Corresponding author

from Frontiers of Retrovirology: Complex retroviruses, retroelements and their hosts

Montpellier, France. 2I-23 September 2009

Published: 24 September 2009

Retrovirology 2009, 6(Suppl 2):P28 doi:10.1 I86/1742-4690-6-S2-P28

This abstract is available from: http://www.retrovirology.com/content/6/S2/P28

(C) 2009 Deroubaix et al; licensee BioMed Central Ltd.

\section{Background}

Most DNA viruses replicate inside the nucleus. In nondividing cells the viral genome enters the nucleus via the nuclear pores. Nuclear import of viral genomes is an active process, which is mediated by a variety of different nuclear localization signals (NLS), being exposed on the surface of genome-associated proteins. Knowledge on viral nuclear transport is very limited as the quantitative impact of the attached viral proteins is often restricted. In cellulo experiments are frequently impaired by the different expression levels of the analyzed proteins making it difficult to quantify the import reaction.

\section{Materials and methods}

The aim of this work is to establish a technique, which allows the quantitative analysis of the karyophilic properties of different proteins or domains of interest in vivo. The system uses confocal microscopy and will be based on a double FRET (fluorescence resonance energy transfer) We use cells, stably expressing interaction partners for the protein of interest (POI). For nuclear detection a YFP labelled karyophilic protein (YFP-NLS) fused to a synthetic a helix is expressed. For cytoplasmic detection a YFP labelled protein with a plasma membrane anchoring domin an a helix is co-expressed (YFP-Mb). The POI is fused to CFP and an a helix interacting with the a helices of YFP-NLS and YFP-Mb. The read-out of the system is the FRET between the POI and partner proteins.

\section{Results}

We first constructed expression vectors and confirmed expression and localization using the YFP fluorescence.
YFP-NLS was imported into the nucleus showing enrichment in defined areas, which represent most likely nucleoli. YFP-Mb properly inserted into the plasma membrane. CFP fused to POI showed nuclear import and colocalization with YFP-NLS. Closed proximity beyond the resolution limit of the microscope was shown was shown by FRET.

\section{Conclusion}

The proposed technique will allow quantifying not only the expression level of individual proteins of interest but as well the quantification of transport via its interaction with the partner proteins. The presented results show the proof of principle of the designed experimental set up. In futur, YFP will be changed to cherry in order to allow the detection of 2 different FRET in the same cell. We assume that the system will allow automated quantification in high through put. 